\title{
Preterm prelabour rupture of membranes
}

\author{
David James
}

\section{The problem}

Unfortunately there are too many examples in obstetrics of very important conditions for which there is no consensus of professional opinion about definition, diagnosis, risks, and above all, management. Preterm prelabour rupture of the membranes (PPROM) is such a case.

The best, most widely used, and simplest definition of PPROM is membrane rupture occurring before the onset of regular uterine contractions prior to 37 weeks' gestation. Some authors add caveats, for example, about the interval between membrane rupture and the onset of labour or not including cases above 34 weeks' gestation. In general, these are unhelpful modifications to the definition. However, while not helpful for definition, these two factorsduration of membrane rupture and gestational age-critically influence the risks and management of PPROM.

The reported incidence of prelabour membrane rupture in all pregnancies varies from 1$10 \%$ depending on the population studied. However, if elective preterm deliveries and fetal death before labour are excluded, the incidence of PPROM in women delivering preterm is between $40-60 \%$. The main independent risk factors for PPROM are antepartum vaginal bleeding, maternal smoking, and previous preterm delivery.

The aetiology and pathogenesis of PPROM are poorly understood. For example, it is not clear whether pregnancies complicated by spontaneous preterm labour and delivery with PPROM should be regarded as different in these respects from cases without preceding membrane rupture. Factors claimed to be possible causes of PPROM include infection, membrane collagen abnormalities, cervical incompetence, raised intrauterine pressure, and low membrane calcium and magnesium content. There have been many theories of the mechanisms whereby these factors produce PPROM with much research currently involving platelet activating factor and prostaglandin metabolism in the membranes. As with most aspects of spontaneous preterm delivery, the explanation remains as elusive as ever.

While the causes of PPROM are uncertain the risks are well known. The three serious complications, in order of importance, are preterm labour and delivery and its consequences, infection, and pulmonary hypoplasia. Other important complications are various deformities due to oligohydramnios, placental abruption, cord compression (either due to cord prolapse or severe oligohydramnios), and mechanical difficulties at delivery. There is no information to indicate whether these risks are any different for 'hindwater' as opposed to 'forewater' PPROM thus in practice no distinction is made between the two.

\section{The problem of diagnosis}

The diagnosis of membrane rupture is not difficult if there is liquor gushing from the vagina or the cervix on sterile speculum examination. However, in many instances this is not the case. A careful history is important, with special care being taken to distinguish PPROM from urinary incontinence. The presence of white flecks (vernix) or green coloration (meconium) of the the liquor may be a useful feature in the history. However, vernix and meconium are characteristic of term liquor and seldom found before 32 weeks. If a sample of fluid is available for examination then it may be possible to decide whether it is liquor. Amniotic fluid contains protein and produces a typical fern like pattern when allowed to dry on a microscope slide; urine does not, but in contrast contains creatinine and uric acid. The $\mathrm{pH}$ of liquor, at $7 \cdot 1-$ $7 \cdot 3$, tends to be higher than that of urine.

A variety of tests have been developed specifically to distinguish liquor not only from urine but also from vaginal secretions and other fluids, but they all have limitations. Some, including the ferning test, are of little value at preterm gestations. For example, the Nile blue test, which produces a positive result by detecting the presence of orange staining anucleate cells in liquor, has a false negative rate of $90 \%$ at 36 weeks and $99 \%$ at 32 weeks. Detection of $\alpha$ fetoprotein using monoclonal antibodies should be more accurate at preterm gestations; however, it has not yet been adequately evaluated in clinical practice. The nitrazine test is probably the most widely used. The underlying principles are that nitrazine solution changes from yellow-green to dark blue at a $\mathrm{pH}$ of 6.5 or more and while vaginal $\mathrm{pH}$ during pregnancy is normally between $4 \cdot 5-6 \cdot 0$ that of liquor is usually alkaline. Unfortunately nitrazine can also turn blue when exposed to tap water, certain antiseptic solutions, cervical mucus, semen, blood, and alkaline urine.

The transabdominal injection of various dyes 
in order to ascertain whether they appear through the cervix as an invasive method of diagnosing membrane rupture is not without risk for the fetus and is not widely practised.

Ultrasound evaluation of liquor volume has merit in theory. The presence of oligohydramnios on scan with a history suggestive of membrane rupture would probably correctly confirm the diagnosis. However, in practice ultrasound is of limited value. To produce appreciable oligohydramnios on ultrasound, the clinical features are usually clear cut with liquor leak continuing and oligohydramnios being obvious on abdominal palpation. In contrast, the commoner clinical dilemma is where there has possibly been a small leak which has stopped. In such cases ultrasound will not detect the small reduction of liquor volume.

\section{The problem of preterm delivery}

Preterm labour and delivery and its complications (respiratory distress syndrome, intraventricular haemorrhage, and chronic lung disease) are the most common and important consequences of PPROM. Approximately $60 \%$ of cases will go into labour within one week of PPROM at 24 weeks' gestation or less; this figure rises to $80 \%$ after 34 weeks.

Because of the difficulties in accurate early diagnosis of intrauterine infection (see below) clinical management has concentrated on trying to assess the consequences of preterm delivery especially when PPROM occurs before 34 weeks. Almost exclusively the evaluation has centred on fetal pulmonary maturity and the likelihood of respiratory distress syndrome. In this regard the traditional lecithin:sphingomyelin ratio in amniotic fluid has proved unreliable, especially when the liquor has been collected vaginally. Fortunately, phosphatidylglycerol is not found in any other body fluids than mature lung surfactant and thus its estimation is the test of choice in such circumstances. Of course, demonstration of fetal lung maturity does not necessarily mean that other consequences of preterm delivery will not occur and that early elective delivery should be automatically undertaken. Arguably the main value of demonstrating fetal surfactant production is that maternal administration of steroids can be avoided (see below). At present the timing of elective delivery in PPROM even with fetal lung maturity is a matter of clinical judgment rather than on the basis of properly conducted randomised trials. In practice, most clinicians tend to be more conservative the more preterm the pregnancy and opt for elective delivery only after 34 weeks.
The problem of infection

The early diagnosis of intrauterine infection in PPROM is important yet difficult. The classical signs of maternal fever, maternal and fetal tachycardia, a tender uterus, and foul smelling liquor are probably relatively late features. Furthermore intrauterine infection is not the only explanation of maternal fever or maternal or fetal tachycardia. Another confounding factor is that the discharge from fulminating aerobic infection may be inoffensive.

A number of laboratory methods have been introduced in an attempt to achieve earlier diagnosis of intrauterine infection with PPROM. In general they have proved unreliable. A summary of the tests is given in the table. Gram stain, white cell count, and culture of the liquor is probably the best of those available. But from where should the liquor be obtained for this bacterial examination? Collecting it vaginally is easier and identifying certain fetal pathogens such as group B streptococci, Escherichia coli, or bacteriodes, especially if single isolates, may be important. However, the usefulness of finding such organisms in planning subsequent management is not known. Negative cultures, on the other hand, are reassuring. Because of this dilemma of possible vaginal commensals contaminating liquor collected via that route, obtaining liquor by amniocentesis has been advocated to allow a more specific diagnosis of intrauterine infection. Unfortunately, there is no evidence to show that this approach is more accurate at diagnosing intrauterine infection. Furthermore, liquor is not obtained in $30-50 \%$ of cases and the procedure carries a risk to the fetus of trauma and the introduction of infection.

Of more recent interest are the observations that early intrauterine infection appears to be associated with disturbances in fetal behavioural physiology. Abnormalities of fetal heart rate monitoring (cardiotocography) and the fetal biophysical profile (concurrent recording of fetal heart rate, breathing, movements, tone, and liquor volume) correlate closely with proved intrauterine sepsis. These non-invasive tests have obvious advantages over amniocentesis. However, at present it is not known whether use of these tests will prove to be helpful in determining the appropriate management and improving fetal outcome.

There is a widely held view that vaginal examinations in PPROM introduces or causes infection. Although there is a reported association of vaginal examinations and the development of sepsis, there are no controlled data to confirm or refute the view that this relationship is causal. There is no evidence as to whether

Accuracy of prediction of intrauterine infection by laboratory tests in published studies (extracted from Ohlsson and Wang ${ }^{+}$). Results are shown as range

\begin{tabular}{lcccc}
\hline Test & Sensitivity & Specificity & $\begin{array}{c}\text { Positive } \\
\text { predictive } \\
\text { value }\end{array}$ & $\begin{array}{c}\text { Negative } \\
\text { predictive } \\
\text { value }\end{array}$ \\
\hline (1) White cell count, band count (\%) & & & $40-75$ & $40-91$ \\
(2) C reactive protein (\%) & $23-80$ & $61-98$ & $10-100$ & $50-100$ \\
(3) Gram stain, white cells, and culture of liquor (\%) & $37-100$ & $44-80$ & $83-98$ & $56-92$ \\
(4) Leucocyte esterase (\%) & $8-83$ & $84-100$ & $4-100$ & $68-92$ \\
(5) Gas chromatography (\%) & $29-93$ & $56-91$ & $20-88$ & $67-96$ \\
\hline
\end{tabular}

Decimal places are omitted for clarity. The definition of 'intrauterine infection'varied. 
digital or speculum examination is better. In general there are two alternative practices. Firstly, a sterile examination is performed on all cases of PPROM at presentation, though there are then differences of opinion as to how frequently examinations should be subsequently repeated. In contrast, other obstetricians maintain that vaginal examinations should be carried out with PPROM only in active labour. There is no evidence to suggest which approach is superior. What is clear, however, is that vaginal examination should only be undertaken to obtain information that is unavailable by other means.

The problem of pulmonary hypoplasia Preterm delivery is numerically the most important complication of PPROM but pulmonary hypoplasia due to oligohydramnios is in many ways more distressing. The prognosis for respiratory distress syndrome in the preterm baby delivering after PPROM and after 28 weeks' gestation is very good with over $90 \%$ survival rates due to measures such as ventilatory support and exogenous surfactant treatment. Pulmonary morphological development can be impaired with very preterm membrane rupture especially before 26 weeks. Thus a mother might spend weeks in hospital, the membranes having ruptured before 26 weeks, only for the baby to be delivered, perhaps after 30 weeks, and dying from pulmonary hypoplasia. Theories of the pathogenesis of the condition include fetal compression reducing lung growth, decreased fetal breathing, and excessive loss of lung fluid.

The problem before birth is that there is no reliable way of diagnosing pulmonary hypoplasia. Fetal breathing movements, chest wall growth, and liquor volume measurements have all been advocated but all have limitations. In practice, an expectant approach is followed even if there is a strong suspicion that the condition may be likely to develop. For example, with membrane rupture early in the second trimester, continued fluid loss, and severe oligohydramnios then pulmonary hypoplasia is highly probable but not every case will result in the problem.

Amnioinfusion (restoration of the amniotic fluid volume with, for example, $\mathrm{N}$-saline) is an alternative approach to the problem currently under evaluation. Although there is evidence that such an approach may reduce caesarean section rates, fetal heart abnormalities, and fetal acid-base status in the presence of PPROM, it is not clear whether the overall outlook for the fetus is any better. The outcome of randomised controlled trials is awaited.

\section{The problem of management}

Since preterm labour and delivery are the main risks of PPROM, referral to a centre with facilities for neonatal intensive care, if these are not available locally, is generally accepted as wise management. There is, however, less agreemen over other aspects of management of PPROM. Furthermore, a review of the published evi- dence from controlled studies is complicated by factors such as the heterogeneous nature of study groups, possible selection bias, small numbers, uncontrolled and variable concomitant treatment. Some generalisations are possible, however.

There is no consistent evidence that the use of prophylactic antibiotics in PPROM reduces maternal infection risk before delivery, increases neonatal sepsis risk after delivery, or significantly prolongs pregnancy. The only proved benefit is that maternal infection rates after delivery are significantly reduced by antibiotic prophylaxis. These observations together with the largely theoretical rather than proved consequences of the development of resistant strains and masking of infection in the newborn are probably the reasons for many authors not supporting the routine use of prophylactic antibiotics in PPROM.

There is no proved benefit from the use of oral tocolytics in PPROM. The few comparative studies that have been published suggest a nonsignificant lower incidence of delivery within 48 hours with their use but any such suggestion of benefit is lost when delivery rates at 10 days after PPROM are compared.

There have been several trials of the use of maternally administered corticosteroids, such as dexamethasone, in PPROM and all have demonstrated a clear advantage of the treatment. Overall, treatment with such agents significantly reduced the risk of development of respiratory distress syndrome by approximately $50 \%$. However, in addition all the studies did demonstrate a trend to slightly higher neonatal sepsis rates, though this was not statistically significant. The conclusion from such work is that the benefits of corticosteroids would seem to outweigh the risks.

Comparative studies have been made of 'active approach' to delivery, namely inducing labour after corticosteroid treatment or as soon as there is evidence of fetal lung maturity, with the 'conservative approach' of waiting to a much later gestation such as 34 weeks before inducing labour or until spontaneous labour occurs. There are consistent trends in the published reports of higher rates of maternal sepsis, caesarean section, neonatal sepsis, respiratory distress syndrome, and neonatal mortality with the 'active' policy of early induction of labour and it would seem that it confers more harm than benefit.

When spontaneous labour occurs after PPROM the correct approach is to allow it to proceed. There is circumstantial evidence to suggest that the event itself may be a manifestation of 'subclinical' intrauterine infection. There is no evidence of benefit to mother or baby from the use of tocolytics in this circumstance. The only exception to this view might be where uterine activity commences at a nonviable gestation such as below 24 weeks. However, there are no studies that have addressed this issue and if this approach is followed because 'there is nothing to lose', great vigilance must be maintained for infection. When spontaneous labour occurs after PPROM and there is evidence of infection delivery must be allowed 
to proceed. The chosen mode of delivery would be that appropriate for any equivalent preterm fetus without PPROM. That said, the issue of which is the best method for delivery of the preterm fetus presenting by the breech is not resolved. Furthermore, there is some evidence that in the presence of infection caesarean section may carry a higher morbidity than vaginal delivery. Though there are no trials of the use of antibiotics before delivery in the presence of infection commonsense argues for their use as the objections are largely theoretical.

\section{Conclusions}

Although there are more divergent opinions over the management of PPROM than the scientific evidence can support, the following general guidelines are suggested:

(1) Accurate diagnosis and gestational age estimation are important at the outset.

(2) Transfer to a tertiary referral centre should be considered if local facilities for neonatal intensive care are not adequate.

(3) Prolongation of the pregnancy to 32-34 weeks (rather than active delivery after steroid treatment or evidence of fetal lung maturity) should be the aim.

(4) Surveillance should be maintained for: (a) intrauterine sepsis-with regular maternal pulse and temperature recordings, fetal heart rate, and behaviour monitoring and regular examination of liquor collected vaginally (Gram stain, leucocyte count and culture); (b) fetal compromise-not just from infection but from cord compression using the same regular moni- toring techniques as for sepsis; and (c) uterine activity.

(5) Corticosteroid administration confers more benefit than harm, but the routine use of tocolytics or prophylactic antibiotics is not recommended.

(6) Vaginal examinations should be kept to a minimum and only be used where the information to be gleaned cannot be obtained by any other means.

(7) Where infection occurs intravenous antibiotics should be used and delivery by the normal route for that stage of pregnancy should be undertaken.

Finally, further research is needed in the following areas:

(1) The relative risks of hindwater versus forewater membrane rupture.

(2) More accurate methods of diagnosing intrauterine infection.

(3) The use of prophylactic antibiotics in a selective manner (for example, with concurrent administration of corticosteroids).

(4) Amnioinfusion.

Suggestions for further reading

1 Keirse MJNC, Ohlsson A, Treffers PE, Kanhai HHH. Prelabour rupture of the membranes preterm. In: Chalmers I, Enkin M, Keirse MJNC eds. Effective care in pregnancy and childbirth. Oxford: Oxford University Press, 1989: and child

2 Kilbride HW, Yeast JD, Thibeault DW. Intrapartum and delivery room management of premature rupture of membranes complicated by oligohydramnios. Clin Perinatol 1989;16:863-88.

3 Ohlsson A. Treatments of preterm premature rupture of the membranes: a meta-analysis. Am f Obstet Gynecol 1990; 162:890-906.

4 Ohlsson A, Wang E. An analysis of antenatal tests to detect infection in preterm premature rupture of the membranes. 